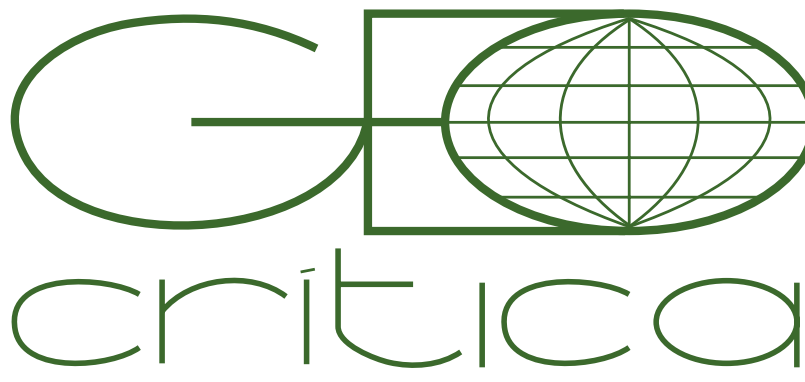

\section{Scripta Nova}

Revista Electrónica de Geografía y Ciencias Sociales Universitat de Barcelona

15 de febrero de 2019

\title{
MODELO PRODUCTOR-INNOVADOR Y AUTOSUFICIENCIA ALIMENTARIA PARA MILPEROS DE SECANO: PROPUESTA DE POLÍTICA PÚBLICA SOSTENIBLE
}

\author{
Miguel Ángel Damián Huato \\ Instituto de Ciencias-BUAP \\ damianhuato@hotmail.com \\ Artemio Cruz León \\ Universidad Autónoma Chapingo \\ etnoagronomia1@gmail.com \\ Dora Ma. Sangerman-Jarquín \\ Instituto Nacional de Investigaciones Forestales, Agrícolas y Pecuarias, Campo Experimental Valle de México, \\ dsangerman@yahoo.com.mx

\section{Lucía López Reyes, Moisés Carcaño Montiel y Omar Romero Arenas* Instituto de Ciencias-BUAP}

\begin{abstract}
Modelo productor-innovador y autosuficiencia alimentaria para milperos de secano: propuesta de política pública sostenible (Resumen)

Se expone una política pública representado por el modelo productor-innovador, donde el maíz se manejó como milpa mediante un diálogo de saberes. Para diseñar el modelo se evaluaron las innovaciones radicales y progresivas aplicadas en el manejo de milpa, donde interaccionaron innovaciones radicales y progresivas, prevaleciendo las segundas y donde casi un tercio de los milperos fueron eficientes, pero los milperos de San Nicolás están más des-campesinados. Si se transfiere el modelo a los milperos menos eficientes, se puede mejorar la autosuficiencia alimentaria.
\end{abstract}

Palabras clave: Milpa, diálogo de saberes, comunidad biótica, descampesinización.

Agradecimientos Al Consejo Nacional de Ciencia y Tecnología y a la Benemérita Universidad Autónoma de Puebla por el

financiamiento otorgado para efectuar esta investigación.

\begin{abstract}
Producer-innovator model and food self-sufficiency for rainfed milperos: sustainable public policy proposal

A public policy is presented, represented by the producer-innovator model, where corn was managed as a milpa through a dialogue of knowledge. In order to design the model, the radical and progressive innovations applied in milpa management were evaluated, where radical and progressive innovations interacted, the latter prevailing and where almost a third of the milperos were efficient, but the milpers of the San Nicolas are more des-campesinados. If the model is transferred to less efficient milperos, food self-sufficiency can be improved.
\end{abstract}

Key words: Milpa, dialogue of knowledge, biotic community, descampesinization. 
Actualmente México vive una crisis estructural originada por la caída de la tasa de ganancia, cuya recuperación exige mayores tasas de explotación del trabajo y de exfoliación de la naturaleza, ocasionando más pobreza alimentaria, la insostenibilidad de las actividades agropecuarias y el cambio climático. Afín con este modelo, se han ejecutado varios programas de Modernización del Campo (1989-1994 hasta 2013-2018), los cuales han eludido mejorar la productividad de los maiceros de secano, porque su producción dejó de formar parte de la reproducción del capital global. Al perder el vínculo con el capital como productor, las políticas públicas los excluyeron de los planes productivos, incluyéndoles en proyectos asistenciales para reproducirlos como fuerza de trabajo y no como productores ${ }^{1}$.

Esta exclusión de los productores de las políticas públicas, contrajo sus rendimientos por hectárea en maíz, principal cultivo de México. Datos del Servicio de Información Agroalimentaria y Pesquera ${ }^{2}$, muestran que durante 35 años (1980-2014) el rendimiento nacional promedio de maíz aumentó $660 \mathrm{~kg}$, al pasar de $1650 \mathrm{~kg}$ (1980) a $2310 \mathrm{~kg} / \mathrm{ha}$ (2014), equivalente a un crecimiento anual de $0.82 \%$.

Estos bajos rendimientos derivaron en mayor importación de maíz amarillo de los Estados Unidos de Norteamérica, pasando de 121 mil toneladas entre 1992-1993 a casi 4 millones en 2003-2004, acelerándose a partir de 2008, hasta alcanzar la cifra actual de más de 12 millones de toneladas ${ }^{3}$. González y $\mathrm{Col}^{4}$, demostraron que se trata de un maíz de baja calidad nutricional con residuos de transgenes y glifosato causando mayor obesidad y desnutrición. Indicaron también que en ninguna de las harinas hechas con maíces nativos estuvo contaminada.

La alimentación es el derecho humano más elemental registrado en la Declaración Universal de Derechos Humanos de las Naciones Unidas; ; condiciona la sobrevivencia de las personas y es esencial para desplegar otros derechos humanos. La agricultura es esencial para el suministro de alimentos y para la satisfacción de las necesidades más básicas del ser humano ${ }^{6}$. Este derecho se va a garantizar cuando se consiga la autosuficiencia alimentaria; es decir, cuando los productores posean la capacidad para satisfacer sus necesidades alimenticias mediante la producción local.

Aumentar los rendimientos y la producción sostenida de maíz en secano técnicamente es viable; el problema radica en que la modernización agrícola ha promovido políticas públicas basadas en la simplificación del manejo de maíz, acotándolo únicamente al uso de agroquímicos, que son sistematizados en paquetes tecnológicos por el INIFAP 7 . Se trata de "recomendaciones generales y/o universales" que engloba tecnologías creadas por la vía experimental, considerada como el único método científico.

\footnotetext{
1 Rubio, 2014.

2 SIAP, 2017.

3 CEDRSSA, 2017, p. 5.

4 González et al., 2017, p. 3-4.

5 ONU, 1948, p. 4.

6 FAO, 2016, p. 4.

7 INIFAP, 2009.
} 
Para De Sousa ${ }^{8}$ se trata de la monocultura del saber reconocida como una sociología de las ausencias: la idea de que el único saber riguroso es el saber científico, y por tanto, otros conocimientos no tienen la validez ni el rigor del conocimiento científico. Pero, si se registran todos los saberes, conocimientos y tecnologías que en la praxis intervienen en el manejo de maíz, encontramos que muchas de ellas forman parte de la sabiduría ancestral de los campesinos, que han jugado un rol trascendental en el desarrollo de las fuerzas productivas.

Este manejo enuncia la forma en cómo el productor combina durante el ciclo agrícola los recursos productivos (tierra, trabajo y tecnología) de los que dispone. Con este fin, ejecuta varias tareas (barbecho, siembra, labores de cultivo, etc.) hechas sucesivamente a nivel de campo, aplicando tecnologías e insumos modernos (híbridos, agroquímicos, etc.) o campesinas (semillas criollas, asociación de cultivos, etc.) o un diálogo de saberes, cuando se aplican tecnologías radicales y progresivas simultáneamente. Este manejo se encuentra muy influido por condiciones de producción endógenas (clima, suelo, flora, etc.) y exógenas (programas de fomento agrícola, ingresos familiares, etc.), inalterables a mediano plazo,

En este manejo destaca la tecnología porque es la palanca más eficaz para mejorar la productividad del trabajo y de la tierra. Innovar significa introducir cambios tecnológicos radicales y/o cambios incrementales o progresivos ${ }^{9}$. Es radical, cuando se aplican prácticas e insumos que habitualmente no han usado los productores o progresiva-incremental, cuando supone la no sustitución radical de toda la estructura tecnológica que se aplica ${ }^{10}$.

El diálogo de saberes es el objetivo fundamental de la investigación agroecológica. La agroecología reconoce (que) en esos lenguajes de larga historia que todavía sobreviven en las mentes y en las manos de los miembros de las culturas rurales, existe un arsenal nemotécnico de un valor inconmensurable ${ }^{11}$. Pero, este diálogo también comprende la aplicación de tecnologías agrícolas modernas, donde ambas, son reconocidas como esenciales para conseguir la autosuficiencia alimentaria y mitigar el cambio climático.

El manejo de maíz secano para los campesinos mexicanos es una cuestión de sobrevivencia, porque la mayor parte de su producción la destina al autoconsumo y porque lo manejan bajo condiciones de secano y en entornos edafo-climáticos y de políticas públicas adversas. Por esta razón, la mayoría de los productores de secano han manejado el maíz como milpa; es decir, asociado con otros cultivos, sobre todo, frijol-calabaza y múltiples arvenses. La milpa es el principal agroecosistema de México y este la unidad básica de estudio de la agroecología, debido a que contiene de modo abstracto, oculto y no desarrollado, todas las determinaciones y especificidades que caracterizan y definen a esta ciencia.

8 De Sousa, 2009.

9 Oslo, 2006, p. 37.

10 Galende, 2008, p. 171.

11 Toledo, 2005: 19. 
Evaluar el manejo de la milpa exige aplicar un enfoque transdisciplinario. Se trata, según Nicolescu ${ }^{12}$ y como el prefijo trans lo muestra, lo que está, a la vez, entre las disciplinas y a través de diversas disciplinas, pero en nuestro caso acotada al manejo de la milpa, que debe ser razonado como totalidad concreta o no sistémica. Así, no se reducen los alcances del estudio a cada practica agrícola, sello de la unidisciplina, pero tampoco se sobrevalora la relación independencia-dependencia de todo con el todo, típico del enfoque sistémico, que segmenta el estudio de la realidad en subsistemas. La totalidad concreta considera la relación que hay entre el manejo de cultivos solamente con ciertas temáticas de otras disciplinas científicas, que son pertinentes para explicar las causas teleológicas de este manejo. Para Zemelma ${ }^{13}$, conocer es especificar y especificar es delimitar relaciones de articulación que posee el problema de estudio respecto de otros fenómenos de la realidad.

Al respecto hay que cuestionarse, sólo para poner un ejemplo: la pluriactividad, un tema propio de la economía, ¿acaso no influye en la especialización que tienen los productores en el manejo de cultivos? Y esta especialización, ¿no se expresa en un manejo eficiente o ineficiente de los mismos? y un manejo eficiente o ineficiente ¿acaso no se manifiesta en una mayor o menor productividad del trabajo y de la tierra y, por ende, en mayores rendimientos por hectárea? Pero no es toda la ciencia económica la que influye en el manejo de los cultivos, sino únicamente algunas de sus aristas que incide y se relaciona con el manejo de la milpa.

Asimismo, en el manejo de la milpa, o de cualquier otro cultivo concurren, desde el nivel local, productores con capacidades productivas diferenciadas, donde destacan los eficientes, cuyo patrón tecnológico fue acreditado como Modelo Productor-Innovador (MP-I). Es un patrón tecnológico donde el maíz se maneja como milpa mediante un diálogo de saberes, donde se funden dos tecnologías, la campesina y la moderna, las cuales son antagónicas y, a la vez, complementarias.La superación dialéctica de una y otra, se sintetizan en un nuevo patrón tecnológico (MP-I) con cualidades tecnológicas superiores que a las que les dieron origen. La eficiencia productiva del MP-I deriva de este diálogo que, a la vez, potencia la eficiencia energética, la estabilidad, la resiliencia y la sostenibilidad de la milpa. Por esta razón el MP-I puede garantizar la autosuficiencia alimentaria a través del tiempo.

La pérdida de saberes y tecnologías campesinas y, por ende, del diálogo de saberes, es un mecanismo que impulsa la descampesinización, que paulatinamente socava su propia cosmovisión. Para Ploeg ${ }^{14}$ la descampesinización se refiere a un debilitamiento, una erosión o incluso la desaparición de prácticas campesinas y a la racionalidad asociada con ellas.

En este estudio se expone una metodología para evaluar el manejo de maíz sembrado como milpa, que sirve como base para diseñar y proponer una política pública basada en el MP-I, para potenciar a nivel local, la producción y consumo de los bienes

12 Nicolescu, 2009, p. 35.

13 Zemelman, 1987

14 Ploeg, 2010, p. 65. 
agrícolas asociados en la milpa, sobre todo de maíz, para alcanzar la autosuficiencia alimentaria, suponiendo un consumo per cápita anual promedio de $500 \mathrm{Kg}^{15}$.

La investigación se realizó en dos municipios del estado de Puebla-México. Cohetzala, situado al Suroeste del estado de Puebla, posee un clima semiseco muy cálido con lluvias en verano; los suelos dominantes son los regosoles que son poco evolucionados, de textura arenosa y con pequeños fragmentos de roca similar al material madre. Se sembraron 1,308 hectáreas, 95\% de secano y el maíz cubrió 98.5\% del área total sembrada. El otro municipio fue San Nicolás de los Ranchos, ubicado en las faldas del Popocatépetl, cerca de la zona Metropolitana de Puebla. Su clima es frío con lluvias en verano e imperan los suelos regosoles. Se cultivaron 2,211 hectáreas de secano y el maíz cubrió 61\% del área total ${ }^{16}$.

\section{Métodos y técnicas de investigación}

La metodología aplicada en el estudio abarcó cuatro fases.

En la primera fase se realizó una encuesta que ae diseñó y aplicó un cuestionario a una muestra de productores adscritos al Programa Directo de Apoyo al Campo (PROCAMPO), con preguntas referidas a las dos condiciones que influyen en el manejo del maíz. El tamaño de la muestra se estimó en $\mathrm{n}=60$ y $\mathrm{n}=77$ maiceros para Cohetzala y San Nicolás, respectivamente ${ }^{17}$.

En la segunda fase, con los datos acopiados por la encuesta se evaluaron las innovaciones radicales y progresivas aplicadas en el manejo del maíz, mediante el cálculo del Índice de Apropiación de Tecnologías Radicales (IATR) y el Grado de Empleo de Tecnologías Progresivas (GETP).

Para calcular el IATR se contrastaron las fórmulas de producción expuestas en el paquete tecnológico generado y recomendado por el INIFAP (Cuadro 1), con la práctica agrícola correspondiente que realizó el productor en el campo. Al paquete se le asignó un valor de 100 puntos y se ponderó, según el impacto de cada componente, en la productividad: 10 fecha de siembra, 20 variedad, 15 densidad de plantas por hectárea, 25 y 5, para dosis de fertilizantes y fecha de su aplicación, 6 y 4, para tipo y dosis de herbicida, 6 y 4, para tipo y dosis de insecticida y 5 para el combate de enfermedades ${ }^{18}$. Cada valor ponderado se dividió entre dos, donde el primer cociente correspondió al uso de la recomendación y el segundo a su adecuado manejo. Por ejemplo, si un productor aplicó la dosis de herbicida recomendada por el INIFAP se le asignó 6 puntos, pero si usó otra dosis el valor fue la mitad y si no usó, su cuantía fue de cero. Esto se debe a que el INIFAP recomienda fórmulas de producción, probadas experimentalmente durante varios años y no el uso de cualquier dosis de herbicida.

15 Warman, 2001.

16 SIAP, 2017.

17 Cochran, 1982: 110

18 INIFAP, 2009. 
Cuadro 1. Paquetes tecnológicos recomendado por el INIFAP para el manejo de maíz en Cohetzala y San Nicolás de los Ranchos (SNR), Puebla-México.

\begin{tabular}{|c|c|c|}
\hline & Práctica/Innovación & Recomendación \\
\hline \multirow{7}{*}{$\begin{array}{l}\frac{\pi}{\pi} \\
\stackrel{N}{N} \\
\frac{0}{0}\end{array}$} & Fecha de siembra & Entre marzo-mayo \\
\hline & Tipo de semilla & $\begin{array}{l}\mathrm{H}-137, \mathrm{H}-139, \mathrm{H}-34, \mathrm{H}-30, \mathrm{H}-33, \mathrm{H}-40, \mathrm{H}-48, \mathrm{H}-50, \mathrm{H}-311, \mathrm{H}-516, \mathrm{H}-515, \mathrm{VS}-536 \\
\mathrm{H}-507, \mathrm{H}-509, \mathrm{~V}-524, \mathrm{VS}-529 \text { y VS-22 }\end{array}$ \\
\hline & Densidad de plantas/ha & 50-60 mil plantas \\
\hline & Fórmula de fertilización & $120-60-00 ; 100-50-00 ; 180-80-60$ \\
\hline & Fecha de fertilización & Se aplica en la siembra y segunda labor \\
\hline & Tipo y dosis herbicida/ha & $\begin{array}{l}\text { Gesaprím } 50(1 \mathrm{Kg}) \text {; } 500 \mathrm{FW}(1.5 \mathrm{~L}) \text {; Gesaprím } 50(1 \mathrm{Kg}) \text { y Hierbamina }(1 \mathrm{~L}) \text {; Basagrán } 480 \\
(0.5 \mathrm{~L}) \text {; Marvel }(1 \mathrm{~L}) \text {; Fitoamina } 2.4 \mathrm{D}(1 \mathrm{~L}) \text {, Hierbamina 2.4 D (1 L); Esterón } 2.4 \mathrm{D}(1 \mathrm{~L}) \text {. }\end{array}$ \\
\hline & Tipo y dosis insecticida/ha & $\begin{array}{l}\text { Volatón al 2.5\% (25 Kg); Volatón 5\% (12 Kg); Furadán 5\% (12 Kg); Folimat } 1000 \text { (0.5 } \\
\text { L); Parathión metílico 50\% (1 L); Malathión (1 L); Sevín } 80 \text { (1 Kg); Sevín 80\% P H (1 Kg); } \\
\text { Malathión } 1000 \text { E (1 L); Diazinón 25\% (1 L). }\end{array}$ \\
\hline \multirow{7}{*}{$\stackrel{c}{\text { 品 }}$} & Fecha de siembra & Entre marzo-mayo \\
\hline & Tipo semilla & $\mathrm{H}-30, \mathrm{H}-33, \mathrm{H}-34, \mathrm{H}-40, \mathrm{H}-48, \mathrm{H}-50 \mathrm{H}-137, \mathrm{H}-139, \mathrm{VS}-22$ \\
\hline & Densidad de plantas/ha & 50 mil plantas \\
\hline & Fórmula fertilización & $140-60-00$ y $110-50-00$ \\
\hline & Fecha fertilización & Durante la siembra y segunda labor \\
\hline & Tipo y dosis herbicida/ha & Gesaprím 50 (1 Kg), 500 FW (1.5 L); Gesaprím 50 (1 Kg) más Hierbamina (1 L). \\
\hline & Tipo y dosis insecticidas/ha & $\begin{array}{l}\text { Volatón } 2.5 \% \text {, Furadán } 5 \% \text { ó Volatón } 5 \%(12-25 \mathrm{Kg} \text { ); Folimat } 1000 \text { (0.5 L); Parathión (1 L) } \\
\text { metílico } 50 \% \text { o Malathión (1 L) disuelto en } 200 \text { L de agua por hectárea. }\end{array}$ \\
\hline
\end{tabular}

Para estimar el IATR se empleó la ecuación número 1.

$$
\operatorname{IATR}=\sum_{\mathrm{i}=1}^{\mathrm{k}}\left[\left(\mathrm{p}_{\mathrm{i}}\right)\left(\mathrm{SPA}_{\mathrm{i}} / \mathrm{PTA}_{\mathrm{i}}\right)\right]
$$

Donde:

IATR: Índice de apropiación de tecnologías radicales.

$\mathrm{K}=10$ : Número de componentes del paquete tecnológico recomendado por el INIFAP;

Pi: Ponderación otorgada al i-ésimo componente de recomendación.

$\sum \mathrm{ki}=1(\mathrm{pi})=100, \mathrm{i}=1,2, \ldots . \mathrm{K}$; SPAi: Sistema productivo agrícola para el i- ésimo componente de recomendación; $\mathrm{i}=1,2, \ldots \mathrm{k}$; PTAi: Paquete tecnológico agrícola para el i -ésimo componente de recomendación; i=1, 2,...k, y (SPAi/PTAi): Proporción de tecnología usada, respecto a la recomendada.

También se calculó el GETP, que incluyó prácticas agrícolas (asociación y rotación de cultivos, conservación de suelo) e insumos (semilla criolla y estiércol) no idóneos para el INIFAP, pero aplicados usualmente por los maiceros, adjudicándoles un valor de 20 unidades a cada una de ellas. El GETP se obtuvo aplicando la ecuación 2.

$$
\mathrm{GETP}=\sum_{\mathrm{i}=1}^{\mathrm{k}} \mathrm{v}_{\mathrm{i}}
$$

Donde:

GETP: Grado de empleo de tecnologías progresivas; K=5: Número de tecnologías progresivas consideradas para el estudio; Vi: Valor asignado a la i- ésima tecnología 
progresiva en función de su uso o no por el productor. El valor fue 0 si el productor no uso la tecnología o 20 si la utilizó

Ya calculados el IATR y el GETP, se construyó la tipología de productores, agrupándolos según el valor del IATR y GETP en baja (< a 33.33), media (33.34-66.66) y alta apropiación de tecnología (> a 66.66). Esta tipología, permitió transitar de las abstracciones empíricas a las constructivas y reflexivas, las cuales son esenciales para la construcción del conocimiento ${ }^{19}$.

En la tercera fase se construyó una tipología de productores según sus rendimientos por hectárea. Con este fin, se eligieron a los productores de mayor y menor rendimiento y se tasó la diferencia, la cual se dividió entre tres y el cociente se sumó al rendimiento menor para crear tres rangos acordes a tipos de productores de bajo, medio y alto rendimiento. Los últimos productores, se consideraron eficientes y su patrón tecnológico se asumió como el MP-I y como propuesta de política pública, ya que su apropiación por parte de los productores menos eficientes, puede aumentar sus rendimientos unitarios de maíz. Esta tipología permitió caracterizar el patrón tecnológico de los maiceros eficientes y analizar los procesos tecnológicos clave generados en el MP-I.

En la cuarta fase se estimó el consumo de maíz familiar real y potencial, considerando el rendimiento (Kg/ha), el área cosechada y número de miembros de que constan las familias, considerando un consumo per cápita de $500 \mathrm{Kg}$. El consumo potencial derivó al considerar que los productores menos eficientes se pueden apropiar del MP-I lo que, hipotéticamente, incrementaría sus rendimientos por hectárea.

\section{Resultados y discusión}

\section{Tipos de productores, apropiación de tecnologías y eficiencia productiva}

Al calcular el IATR (Cuadro 2) se halló que el uso de estas innovaciones fueron en promedio bajo: 26.7 unidades para Cohetzala y 40.6 para San Nicolás, encontrándose productores sólo de baja y media apropiación de tecnologías modernas. Además fueron diferenciadas, con un rango que va de 22.1-37.3 unidades de Cohetzala y para San Nicolás de 28.3-44.1, expuestos en el mismo orden. Por otra parte no se halló relación entre el uso de estas tecnologías y el rendimiento.

Por ejemplo, en Cohetzala hubo 15.2 unidades de diferencia en el IATR entre maiceros de baja y media apropiación, que no se expresó en mayor rendimiento ya que obtuvieron, respectivamente, 745 y $748 \mathrm{Kg} / \mathrm{ha}$. En San Nicolás esta diferencia entre maiceros de baja y media apropiación fue un poco mayor (15.8 unidades), pero los primeros tuvieron mayores rendimientos por hectárea (1359 Kg) que los segundos (1343 Kg).

La discordancia que hay entre el uso de innovaciones radicales y los rendimientos, se debe a que el manejo moderno aplica agroquímicos, en agronichos que tienen suelos degradados y un ciclo lluvias errático, que per se no garantizan mayor productividad.

19 Piaget y García, 2008, p. 193. 
Cuadro 2. Número de productores, IATR y rendimiento por tipo de productores de Cohetzala (C) y San Nicolás de los Ranchos (SNR), Puebla-México.

\begin{tabular}{|c|c|c|c|c|c|c|c|}
\hline & \multirow{2}{*}{ MP-Ios/Indicador } & \multicolumn{2}{|c|}{ Baja } & \multicolumn{2}{|c|}{ Media } & \multicolumn{2}{|c|}{ Prom. Mpal. } \\
\hline & & Núm. & $\%$ & Núm. & $\%$ & Núm. & $\%$ \\
\hline \multirow{3}{*}{$\cup$} & Productores & 42 & 70 & 18 & 30 & 60 & 100 \\
\hline & IATR & \multicolumn{2}{|c|}{22.1} & \multicolumn{2}{|c|}{37.3} & \multicolumn{2}{|c|}{26.7} \\
\hline & Rendimiento* & \multicolumn{2}{|c|}{$745^{a}$} & \multicolumn{2}{|c|}{$748^{a}$} & \multicolumn{2}{|c|}{746} \\
\hline \multirow{3}{*}{ 孚 } & Productores & 17 & 22 & 60 & 78 & 77 & 100 \\
\hline & IATR & \multicolumn{2}{|c|}{28.3} & \multicolumn{2}{|c|}{44.1} & \multicolumn{2}{|c|}{40.6} \\
\hline & Rendimiento* & \multicolumn{2}{|c|}{$1359^{a}$} & \multicolumn{2}{|c|}{$1343^{a}$} & \multicolumn{2}{|c|}{1347} \\
\hline
\end{tabular}

Fuente: Elaboración propia con datos obtenidos de la encuesta, 2009.

* Letras iguales en las medias de rendimiento, evidencia que no hay diferencia estadística significativa entre ellas (Prueba t de Student, $\mathrm{p}<0.05)$.

Por tanto, no fue casual que al medir el GETP (Cuadro 3) se evidenciara que en el manejo de maíz predominaran las innovaciones progresivas sobre las radicales: en Cohetzala el GETP fue mayor 42.6 unidades que el IATR y en San Nicolás 20.7. El GETP también fue diferenciado: en Cohetzala el rango fluctuó entre 57.5-83 unidades para milperos de medio y alto GETP, y en San Nicolás, estas cifras fueron de 20-5482 unidades para maiceros de bajo, medio y alto rendimiento. Además, hubo una relación directa entre GETP y rendimientos.

Cuadro 3. Número de productores, GETP y rendimiento (Kg ha-1) por tipo de milperos de Cohetzala (C) y San Nicolás de los Ranchos (SNR), Puebla-México.

\begin{tabular}{|c|c|c|c|c|c|c|c|c|c|}
\hline \multirow{2}{*}{\multicolumn{2}{|c|}{ MP-los/Indicador }} & \multicolumn{2}{|c|}{ Baja } & \multicolumn{2}{|c|}{ Media } & \multicolumn{2}{|c|}{ Alta } & \multicolumn{2}{|c|}{ Prom. Mpal. } \\
\hline & & Núm. & $\%$ & Núm. & $\%$ & Núm. & $\%$ & Núm. & $\%$ \\
\hline \multirow{3}{*}{$u$} & Productores & 0 & 0 & 32 & 53 & 28 & 47 & 60 & 100 \\
\hline & GETP & \multicolumn{2}{|c|}{--- } & \multicolumn{2}{|c|}{59} & \multicolumn{2}{|c|}{83} & \multicolumn{2}{|c|}{70} \\
\hline & Rendimiento* & \multicolumn{2}{|c|}{---} & \multicolumn{2}{|c|}{$704^{a}$} & \multicolumn{2}{|c|}{$793^{b}$} & \multicolumn{2}{|c|}{746} \\
\hline \multirow{3}{*}{ 品 } & Productores & 6 & 8 & 31 & 40 & 40 & 52 & 77 & 100 \\
\hline & GETP & \multicolumn{2}{|c|}{20} & \multicolumn{2}{|c|}{54} & \multicolumn{2}{|c|}{82} & \multicolumn{2}{|c|}{66} \\
\hline & Rendimiento* & \multicolumn{2}{|c|}{$767 a$} & \multicolumn{2}{|c|}{$1358^{b}$} & \multicolumn{2}{|c|}{$1425^{c}$} & \multicolumn{2}{|c|}{1347} \\
\hline
\end{tabular}

Fuente: Elaboración propia con datos obtenidos de la encuesta, 2009.

* Letras distintas en las medias de rendimiento, indica que hay diferencia estadística significativa entre ellas (Prueba t de Student Cohetzala y Prueba de Tukey SNR, $\mathrm{p}<0.05$ )

La evaluación de las tecnologías aplicadas en el manejo del maíz y la agrupación de productores de acuerdo a su productividad, permitió identificar el MP-I, el cual representa nuestra propuesta de política pública para innovar el manejo de la milpa y coadyuvar a mejorar la autosuficiencia alimentaria a nivel local.

\section{Patrón tecnológico de los milperos eficientes y diálogo de saberes}

Para identificar el MP-I se eligieron los rendimientos mayor y menor de los milperos de cada municipio: 400-1000 para Cohetzala y 500-2200 para San Nicolás; se calculó la diferencia, 600 para Cohetzala y 1700 para San Nicolás; se dividió entre tres, dando 
un valor de 200 para Cohetzala y 567 para San Nicolás. Este cociente al sumarse al rendimiento menor, permitió crear tres rangos, referidos a tres tipos de maiceros clasificados según su productividad. Para Cohetzala los rangos fueron: bajos < a 600, medios entre 601-800 y altos > a $800 \mathrm{Kg}$ por hectárea, y para San Nicolás fueron: bajos < a 1067, medios 1068-1635 y altos > a 1635.

La apropiación de tecnologías y los rendimientos de los tipos de milperos se registraron en el cuadro 4, donde se nota que casi un tercio fueron eficientes con rendimientos significativamente mayores que los no eficientes. Por otra parte, los valores del IATR y del GETP se reducen entre los milperos de alta apropiación tecnológica (MP-I) pero, a la vez, su rendimiento es superior debido, quizás, a que ambas tecnologías interaccionan en el diálogo de saberes.

Cuadro 4. Número de productores, IATR, GETP y rendimientos (Kg ha-1), según su potencial productivo de los maiceros de Cohetzala (C) y San Nicolás de los Ranchos (SNR), Puebla-México.

\begin{tabular}{|c|c|c|c|c|c|c|c|c|c|}
\hline & \multirow{2}{*}{ MP-Ios/Indicador } & \multicolumn{2}{|c|}{ Baja } & \multicolumn{2}{|c|}{ Media } & \multicolumn{2}{|c|}{ Alta } & \multicolumn{2}{|c|}{ Prom. Mpal. } \\
\hline & & Núm. & $\%$ & Núm. & $\%$ & Núm. & $\%$ & Núm. & $\%$ \\
\hline \multirow{4}{*}{$u$} & Productores & 14 & 23 & 27 & 45 & 19 & 32 & 60 & 100 \\
\hline & IATR & \multicolumn{2}{|c|}{23.4} & \multicolumn{2}{|c|}{27.3} & \multicolumn{2}{|c|}{28.3} & \multicolumn{2}{|c|}{26.7} \\
\hline & GETP & \multicolumn{2}{|c|}{58.6} & \multicolumn{2}{|c|}{73.3} & \multicolumn{2}{|c|}{71.6} & \multicolumn{2}{|c|}{69.3} \\
\hline & Rendimiento & \multicolumn{2}{|c|}{$486^{a}$} & \multicolumn{2}{|c|}{$751^{\mathrm{b}}$} & \multicolumn{2}{|c|}{$930^{c}$} & \multicolumn{2}{|c|}{746} \\
\hline \multirow{4}{*}{ 品 } & Productores & 27 & 35 & 28 & 36 & 22 & 29 & 77 & 100 \\
\hline & IATR & \multicolumn{2}{|c|}{44.1} & \multicolumn{2}{|c|}{35.8} & \multicolumn{2}{|c|}{42.3} & \multicolumn{2}{|c|}{40.6} \\
\hline & GETP & \multicolumn{2}{|c|}{48.1} & \multicolumn{2}{|c|}{64.3} & \multicolumn{2}{|c|}{73.6} & \multicolumn{2}{|c|}{61.3} \\
\hline & Rendimiento & \multicolumn{2}{|c|}{$763^{a}$} & \multicolumn{2}{|c|}{$1438^{b}$} & \multicolumn{2}{|c|}{$1971^{c}$} & \multicolumn{2}{|c|}{1347} \\
\hline
\end{tabular}

Fuente: Elaboración propia con datos obtenidos de la encuesta, 2009. Dentro de cada hilera (rendimiento, IATR y GETP), letras distintas en las medias indica que hay diferencia estadística significativa entre ellas (Prueba de Tukey, $\mathrm{p}<0.05$ ).

\section{Patrón tecnológico de milperos versus paquete tecnológico del INIFAP}

Al comparar el patrón tecnológico aplicado por los milperos (Cuadro 5), con el que recomienda el INIFAP (Cuadro 1), se advierte que el paquete tecnológico del INIFAP no reconoció ninguna tecnología campesina, a pesar de la enorme importancia que tienen en el manejo de la milpa, proscribiendo saberes que forman parte de la herencia cultural del campesino, y promoviendo la colonización del saber y la violencia epistémica. En cambio, el patrón tecnológico de los milperos, sobre todo el MP-I, reconoció y revaloró los saberes campesinos aplicados en el manejo de la milpa. Además, las fechas de siembra recomendadas por el INIFAP son pertinentes para el Altiplano Poblano donde se ubica San Nicolás, pero no para Cohetzala que tienen un periodo de lluvias bien definido, el cual inicia a fines de junio o principios de julio.

El INIFAP recomienda la siembra de híbridos y todos los maiceros utilizaron semillas criollas porque: las prefieren para elaborar tortillas; tienen una adaptación milenaria a los agronichos locales afectados por siniestros recurrentes; tienen menor costo y una productividad estable en el tiempo; además, son pilares de la reproducción ganadera al proveer de mayor cantidad y calidad de forraje. La densidad de 
plantas por hectárea es mayor que la recomendada, sobre todo en San Nicolás, debido a que abonaron sus suelos con una mezcla de estiércol y fertilizantes sintéticos, que potenciaron la fertilidad de los suelos agrícolas.

El agroquímico más utilizado fue el fertilizante, aunque en dosis menores que las recomendadas por el INIFAP, aun cuando el tipo de suelos (regosoles) que predominan, sobre todo en Cohetzala, son de formación incipiente y de baja fertilidad.

Cuadro 5. Innovaciones utilizadas en el manejo del maíz por los productores según su eficiencia productiva de Cohetzala y San Nicolás de los Ranchos, Puebla-México.

\begin{tabular}{|c|c|c|c|c|c|}
\hline \multirow[t]{2}{*}{ MP-Io } & \multirow[t]{2}{*}{ Dato/Innovación } & Baja & Media & Alta & Prom. Mpal. \\
\hline & & $\%$ & $\%$ & $\%$ & $\%$ \\
\hline \multirow{14}{*}{$\begin{array}{l}\frac{\pi}{0} \\
\mathbb{N} \\
0 \\
0 \\
0 \\
0\end{array}$} & Área sembrada maíz (Ha) & 29.5 & 61 & 47 & 137.5 \\
\hline & Conservación suelos & 14 & 36 & 23 & 27 \\
\hline & Fecha siembra junio & 66 & 48 & 43 & 50 \\
\hline & Fecha siembra julio & 34 & 52 & 57 & 50 \\
\hline & Semilla criolla & 24 & 44 & 32 & 98 \\
\hline & Densidad de plantas (Número) & 51,282 & 53,034 & 50,781 & 51,911 \\
\hline & Asociación de cultivos & 93 & 100 & 100 & 99 \\
\hline & Asociación con leguminosas & 83 & 66 & 100 & 81 \\
\hline & Asociación con calabaza & 63 & 66 & 98 & 76 \\
\hline & Rotación de cultivos & 27 & 46 & 45 & 41 \\
\hline & Aplicación estiércol (Kg/Ha) & 1,434 & 1,677 & 1,775 & 1,644 \\
\hline & Aplicación fertilizantes & 83 & 93 & 100 & 93 \\
\hline & Aplicación herbicidas & 29 & 18 & 15 & 19 \\
\hline & Aplicación insecticida & 0 & 9 & 19 & 11 \\
\hline \multirow{15}{*}{ 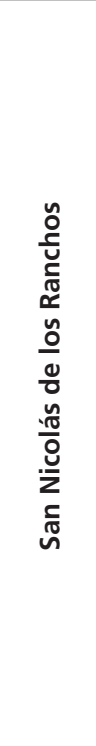 } & Área sembrada maíz (Ha) & 62.25 & 77 & 79.5 & 218.75 \\
\hline & Conservación suelos & 81 & 69 & 61 & 70 \\
\hline & Fecha siembra febrero & 0 & 1 & 0 & 0 \\
\hline & Fecha siembra marzo & 66 & 75 & 71 & 71 \\
\hline & Fecha siembra abril & 0 & 0 & 29 & 11 \\
\hline & Semilla criolla & 97 & 100 & 100 & 99 \\
\hline & Densidad de plantas (Número) & 57,356 & 63,623 & 60,966 & 60,667 \\
\hline & Asociación de cultivos & 31 & 34 & 31 & 32 \\
\hline & Asociación con leguminosas & 3 & 32 & 26 & 30 \\
\hline & Asociación con calabaza & 0 & 0 & 3 & 1 \\
\hline & Rotación de cultivos & 48 & 64 & 84 & 67 \\
\hline & Aplicación estiércol (Kg/Ha) & 550 & 590 & 1,383 & 803 \\
\hline & Aplicación fertilizantes & 96 & 79 & 96 & 90 \\
\hline & Aplicación herbicidas & 85 & 36 & 52 & 56 \\
\hline & Aplicación insecticida & 43 & 14 & 25 & 26 \\
\hline
\end{tabular}

Fuente: Elaboración propia con datos obtenidos de la encuesta, 2009

Otro agroquímico usual es el herbicida sustituto de mano de obra. La exclusión de los maiceros de las políticas públicas acrecentó la emigración y senectud de los maiceros. Del total de miembros que integraron a las familias de los productores de 
Cohetzala (328 personas), 47\% emigraron y su edad promedio fue de 55.5 años. Estos datos para San Nicolás fueron, respectivamente, 434, 20\% y 54.5. El agroquímico menos utilizado fue el insecticida. Herrera ${ }^{20}$ y Col. refieren que la riqueza y abundancia de la artropodofauna es mayor en policultivos que en monocultivos la cual ha sido la base para la creación de cadenas y redes tróficas y, del equilibrio biológico, lo que reduce el perjuicio que ocasionan las plagas.

\section{Patrón tecnológico de los milperos eficientes y agroecología}

Todos los datos encontrados (Cuadro 3, 4 y 5), evidencian que el maíz se maneja como milpa mediante un diálogo de saberes, de donde derivó la mayor productividad de los milperos eficientes, habilitada por cuatro procesos tecnológicos clave que actúan a la par, y que se desagregan únicamente por cuestiones de análisis.

El primero proviene de la comunidad biótica que tiene la ventaja de la co-existencia de plantas C4 y C3 con necesidades distintas de energía solar y de dióxido de carbono lo que eficientiza el uso de estos elementos y la captura de carbono para mitigar el cambio climático. En cambio, el manejo de maíz como monocultivo se caracteriza porque no toda su estructura foliar elabora carbohidratos homogéneamente: cerca del 50\% de los carbohidratos acumulados en el grano de maíz son producidos por las hojas del tercio superior de planta, el 30\% por las del tercio medio, y el $20 \%$ restante, por las hojas ubicadas en la parte basal ${ }^{21}$. Además, la riqueza y abundancia de artropodofauna, auspiciada por la diversidad de plantas que hay en la milpa, re-crea redes tróficas que autorregulan la población de artrópodos presentes en el agroecosistema, lo que deriva en un equilibrio biológico que se logra cuando los patógenos que pueden afectar a las plantas cultivadas son expuestos al ataque de sus enemigos naturales. Por otra parte, la milpa provee más biomasa que se reintegra al suelo, la cual es reciclada por una gran diversidad de microorganismos en nutrientes y energía, para que puedan ser consumidos por los organismos vivos presentes en la comunidad biótica. Finalmente, reproducen especies redundantes que crean redundancia de relaciones, garantizando la estabilidad, la resiliencia y la sostenibilidad en el tiempo de la milpa ${ }^{22}$.

El segundo proceso tecnológico clave deriva directamente de la cooperación que se da entre las plantas asociadas en la milpa. Por ejemplo, el frijol fija nitrógeno atmosférico, elemento básico para la nutrición de las plantas; por su parte el maíz le sirve de tutor al frijol enredador, y la calabaza, con su amplio follaje y hábito rastrero, salvaguarda al suelo de la erosión, fomenta la filtración de agua e impide su evaporación y el crecimiento de arvenses durante los primeros ciclos fenológicos del maíz y frijol.

El tercer proceso deriva directamente de las innovaciones campesinas. La adaptación milenaria de las semillas criollas a los agronichos locales y su estabilidad

20 Herrera et al., 2005, p. 25.

21 Magalhanes y Da Silva; 1987; Fassio et al., 1998.

22 Walker, 1992. 
productiva, se debe a su alta variabilidad que está en permanente cambio ${ }^{23}$. La rotación de cultivos mejora las propiedades de los suelos, retiene humedad y nutrientes, aporta nutrientes y sanidad al suelo. La conservación de suelos evita la pérdida de nutrientes y agua, recursos fundamentales que potencian la productividad. El estiércol es un indicador clave de la calidad del suelo: provee nutrientes, mejora la estructura y textura del suelo, aumenta la aireación, la penetración y la retención de agua, estimula el desarrollo de microorganismos benéficos para la planta y promueve la captura de carbono ${ }^{24}$.

El cuarto proceso proviene de la complementariedad que se da entre las tecnologías campesinas y modernas: por ejemplo, la aplicación de fertilizantes nitrogenados. El nitrógeno es esencial para el crecimiento de plantas, pero también garantiza la diversidad y abundancia de flora y fauna microbiana la cual descompone la materia orgánica. Si ésta tiene alto contenido de nitrógeno, los microorganismos tendrán suficiente sustrato para inducir mayor mineralización, pues la microflora (bacterias, hongos, etc.) tendrá plenamente satisfechas sus necesidades de nitrógeno ${ }^{25}$.

Vistos desde otro ángulo los datos del cuadro 5 indican que los milperos de San Nicolás tienden a modernizar el manejo de maíz, ya que usaron más innovaciones radicales que progresivas. Aplicaron 3.5 más de herbicida y 1.3 más de insecticida que los de Cohetzala, los cuales aplicaron únicamente folidol, insecticida utilizado para combatir a la hormiga arriera (Atta mexicana), una de las plagas más dañinas de "La Mixteca Baja" de Puebla-México, región donde se ubica Cohetzala.

El uso de herbicida en San Nicolás afectó a la asociación de cultivos que fue realizada por una tercera parte de los productores comparados con los de Cohetzala. Como se sabe, el herbicida aniquila a plantas de hojas anchas (frijol, calabaza), pero no a las gramíneas (maíz). Con la pérdida de la comunidad biótica se destruyen las colaboraciones, interacciones y sinergias, promovidos por una de las fuentes vitales del diálogo de saberes y de la productividad. La mengua del policultivo causó que los milperos de San Nicolás realizaran más conservación de suelos, papel que ha cumplido la calabaza en la Mixteca Baja Poblana.

Es decir, los milperos de San Nicolás están en proceso de descampesinización, fenómeno típico del capitalismo, alentado por la cercanía que tienen con la zona metropolitana de Puebla $(40 \mathrm{Km})$, lo cual facilita su incorporación al mercado de trabajo formal e informal, donde realizan tareas que tienen poca relación con el manejo de la milpa. No obstante, creemos que estos trabajadores-milperos seguirán sembrando maíz porque de él depende, en parte, su alimentación, y porque así evitan una mayor extracción de excedentes económicos al prescindir, por un tiempo, de la compra en los mercados locales de este grano, de tortilla, de masa y otros derivados del maíz.

23 Flores y Sarandón, 2014.

24 Robert, 2002, p. 5.

25 Ferrera y Alarcón, 2001. 


\section{Características de los milperos}

Con los datos acopiados de la encuesta estudiamos las características de los tipos de productores, para considerar la viabilidad de transferir el MP-I a los productores menos

eficientes.

Cuadro 6. Características de los productores, según el rendimiento de Cohetzala y San Nicolás de los Ranchos, Puebla-México.

\begin{tabular}{|c|c|c|c|c|c|}
\hline & Municipios/Indicadores & Baja & Media & Alta & Prom. Mpal. \\
\hline \multirow{16}{*}{$\begin{array}{l}\frac{\pi}{\pi} \\
N \\
0 \\
0 \\
0\end{array}$} & Edad & 54.8 & 57.8 & 52.6 & 55.5 \\
\hline & Migrantes/familia (Prom.) & 2 & 3 & 2.4 & 2.6 \\
\hline & Remesas (\$promedio/mes/per cápita) & 475 & 607 & 474 & 534 \\
\hline & Gasto prom. (\$/mes/per cápita) & 927 & 1,132 & 1,001 & 1,043 \\
\hline & Autoconsumo de maíz (\%) & 100 & 89 & 68 & 83 \\
\hline & Autoconsumo y venta de maíz (\%) & 0 & 11 & 32 & 17 \\
\hline & Pluriactivos primarios $^{\mathrm{a}}(\%)$ & 43 & 67 & 95 & 70 \\
\hline & Pluriactivos secundarios ${ }^{\mathrm{b}}(\%)$ & 29 & 15 & 5 & 15 \\
\hline & Maiceros ${ }^{c}(\%)$ & 28 & 18 & 0 & 15 \\
\hline & Área sembrada maíz (promedio ha) & 2.1 & 2.3 & 2.5 & 2.3 \\
\hline & Área de traspatio ( $\mathrm{M}^{\mathrm{b}}$ promedio) & 409 & 515 & 317 & 427 \\
\hline & Recolección de bienes (\%) & 100 & 89 & 89 & 92 \\
\hline & Posesión de tractor (\%) & 14 & 15 & 5 & 7 \\
\hline & Posesión de yunta (\%) & 71 & 70 & 84 & 75 \\
\hline & Ganado mayor (núm. cabezas/prom.) & 4.5 & 6.1 & 5.8 & 5.6 \\
\hline & Ganado menor (núm. cabezas/prom.) & 10.6 & 18.2 & 19.3 & 16.8 \\
\hline \multirow{16}{*}{ 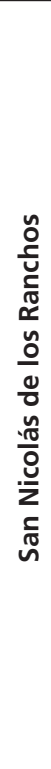 } & Edad & 58.5 & 52.4 & 52.1 & 54.5 \\
\hline & Migrantes/familia (Prom.) & 1.1 & 1.1 & 1 & 1.1 \\
\hline & Remesas (\$/mes/per cápita) & 112 & 94 & 91 & 100 \\
\hline & Gasto prom. (\$/mes/per cápita) & 726 & 657 & 648 & 677 \\
\hline & Autoconsumo de maíz (\%) & 78 & 36 & 9 & 43 \\
\hline & Autoconsumo y venta de maíz (\%) & 22 & 64 & 91 & 57 \\
\hline & Pluriactivos primarios ${ }^{\mathrm{a}}(\%)$ & 15 & 64 & 100 & 57 \\
\hline & Pluriactivos secundarios ${ }^{\mathrm{b}}(\%)$ & 70 & 25 & 0 & 34 \\
\hline & Maiceros` (\%) & 15 & 11 & 0 & 9 \\
\hline & Área sembrada maíz (promedio ha) & 2.3 & 2.8 & 3.6 & 2.8 \\
\hline & Área de traspatio ( $\mathrm{M}^{\mathrm{b}}$ promedio) & 191 & 197 & 356 & 240 \\
\hline & Recolección de bienes (\%) & 96 & 93 & 100 & 95 \\
\hline & Posesión de tractor (\%) & 7 & 7 & 18 & 10 \\
\hline & Posesión de yunta (\%) & 43 & 54 & 77 & 57 \\
\hline & Ganado mayor (núm. cabezas/prom.) & 1.2 & 2.2 & 6.9 & 3.2 \\
\hline & Ganado menor (núm. cabezas/prom.) & 9.8 & 12.4 & 23.3 & 14.6 \\
\hline
\end{tabular}

Fuente: elaboración propia con datos obtenidos de la encuesta, 2009.

a Cultivaron maíz y realizaron otras tareas en ramas económicas del sector primario.

b Ejecutaron otras tareas en sectores secundario y terciario. c Sólo sembraron maíz.

Los rasgos de los milperos se exponen en el Cuadro 6, observándose que tienen condiciones de vida similares: son minifundistas extremos y tienen baja disponibilidad a los medios de producción; la producción se destina preferentemente al autoconsumo, los gastos familiares de los productores se encuentra considerablemen- 
te subsidiados por las remesas, principalmente en Cohetzala, y realizan múltiples estrategias de sobrevivencia predominando la venta de fuerza de trabajo, el manejo de traspatio y de ganado mayor y menor, así como la recolección de bienes naturales.

Destaca, además, que los productores eficientes han diversificado sus tareas económicas en el sector primario y los otros en tareas secundarias y terciarias, causando un aumento de su ingreso ${ }^{26}$, pero, al mismo tiempo, una reducción de su renta agrícola ${ }^{27}$. La práctica de varias actividades se expresa en una menor especialización en el manejo de la milpa y, por ende, en una regresión tecnológica, que será mayor en aquellos productores que efectuaron actividades que tienen poca relación con el manejo de cultivos. A la vez, una mayor regresión tecnológica se va a mostrar en menores rendimientos unitarios

\section{La autosuficiencia alimentaria: producción y consumo de maíz real y potencial}

La alimentación es el cimiento de la vida individual y social; ésta se alcanza por razón de la producción y consumo sostenido de alimentos, que se puede garantizar si somos capaces de incrementar los rendimientos unitarios a nivel local. El maíz ha sido, y es, esencial para la alimentación de los mexicanos, donde cada persona consume, en promedio, 333.6 Kg anual per cápita ${ }^{28}$.

A través de los datos obtenidos de la encuesta, se calculó el consumo real de maíz encontrándose que cada miembro familiar de Cohetzala dispone en promedio al año de $339 \mathrm{Kg}$ de maíz y los de San Nicolás de $726 \mathrm{Kg}$. El 15 y 57\% de personas de Cohetzala y San Nicolás, respectivamente, alcanzaron una producción per cápita de $500 \mathrm{Kg}$, alcanzando la autosuficiencia alimentaria. Cada persona de Cohetzala que dispone de autosuficiencia alimentaria, produce un excedente de $231 \mathrm{Kg}$ por año y en San Nicolás de $767 \mathrm{Kg}$, que venderían en los mercados local y/o regional. La venta de este excedente, es un eslabón económico fundamental para que las familias obtengan otros recursos que destina, en parte, para adquirir otros alimentos y diversificar su alimentación. En contraste, los que no disponen de una producción de $500 \mathrm{Kg}$ en Cohetzala tienen que adquirir en el mercado $229 \mathrm{Kg}$ al año de maíz del que consumen y los de San Nicolás $253 \mathrm{Kg}$. Si los milperos menos eficientes de Cohetzala se apropiaran del MP-I, hipotéticamente los rendimientos por hectárea crecerían, en promedio, 91\% para los productores de bajo rendimiento y $24 \%$ para los de medio. Estas cifras para San Nicolás serían de 150 y $38 \%$, expuestos en el mismo orden.

Al estimar el consumo potencial de maíz calculado con los nuevos rendimientos, se encontró que el consumo per cápita de maíz en Cohetzala se elevaría en promedio a $415 \mathrm{Kg}$ de maíz y en San Nicolás a 1035 Kg. El 27 y 81\% de personas de Cohetzala y San Nicolás respectivamente, dispondrían de autosuficiencia alimentaria de maíz. El déficit de $231 \mathrm{Kg}$ para alcanzar autosuficiencia alimentaria, descendería en Cohetzala a $189 \mathrm{Kg}$ y en San Nicolás a 227 kilos.

26 De Grammont, 2009, p. 274.

27 Anseeuw y Laurent, 2007, p. 2.

28 SIAP, 2017. 
Otras experiencias agroecológicas exitosas, avalan estos resultados ${ }^{29}$. Estos autores evaluaron 40 proyectos de 20 países africanos donde se intensificó el manejo de cultivos durante 2001-2010, mediante el mejoramiento de las cosechas, la lucha integrada contra plagas, la conservación de suelos y la agroforestería. En 2010, el rendimiento medio se multiplicó por 2.13 e incrementó la producción total de alimentos en 5.8 millones de toneladas al año, equivalente a $557 \mathrm{Kg}$ por familia/año.

El Movimiento Campesino a Campesino ${ }^{30}$ aumentó los rendimientos del maíz de media tonelada por hectárea a tres, como resultado de fabricar composta, de hacer rotaciones con leguminosas e intercalar nuevos cultivos en las áridas parcelas de Vicente Guerrero del municipio de Españita, Tlaxcala-México. En el faro agroecológico instaurado por Damián ${ }^{31}$ en Jolalpan, Puebla-México se cosecharon, durante el ciclo agrícola verano-otoño, 2015, $241 \mathrm{Kg} /$ ha de maíz cuando se manejó como monocultivo y $1300 \mathrm{Kg} / \mathrm{ha}$ en policultivo. Además, en la milpa se cosecharon 34.4 y $24.3 \mathrm{Kg} / \mathrm{ha}$ de frijol y calabaza, respectivamente, alimentos básicos de la dieta de las familias campesinas. Estos contrastantes rendimientos fueron alentados por la sequía que afectó al país durante el ciclo referido, y que en Jolalpan tuvo una duración de 49 días.

\section{Modelo Productor-Innovador: propuesta de política pública}

Las políticas públicas se fundamentan en el principio de que el Estado y los poderes políticos son responsables de promover el desarrollo socio-económico para eliminar las desigualdades sociales. Para Patrouilleau ${ }^{32}$, las políticas públicas competen a los instrumentos y estrategias institucionales que desde el Estado se diseñan e implementan con distintos grados de participación de actores de la sociedad civil y de organismos internacionales, con el fin de orientar la ejecución de las acciones de gobierno.

Para revertir los bajos rendimientos de los productores menos eficientes y alcanzar la autosuficiencia alimentaria, se propone que se adopte como política pública el MP-I. Se trata de un manejo innovador de la milpa que está disponible entre los productores de maíz a nivel local. El reto consiste en identificarlo y transferirlo para fortalecer la producción y consumo de maíz a nivel local. Se trata de una alternativa posibilista ya que la mayoría de ellos han manejado el maíz como milpa. Además, esta propuesta resulta viable de ejecutarse porque en México existe el programa institucional Modernización Sustentable de la Agricultura Tradicional (Masagro), que tiene como propósito, aumentar la eficiencia y la productividad de los productores de escasos recursos y garantizar la seguridad alimentaria de una población mundial creciente. Además, los gobiernos de las entidades tienen los recursos humanos y la infraestructura para diseñar y ejecutar este método; además, cuentan con estaciones de radio y televisión, que ya han sido utilizados con éxito para la transferencia de tecnologías. El deterioro de la sinergia agricultura-ganadería y la pérdida de estiércol es

29 Pretty et al. 2011

30 Holt, 2008, p. 24.

31 Damian, 2015, p. 1-2.

32 Patrouilleau et al. 2017, p. 20. 
una enorme oportunidad para promover el uso de desechos orgánicos como abonos, propuesta que fue hecha por Justus von Liebig desde mediados del siglo XIX. Según el SNIARN ${ }^{33}$ en 2012 se generaron 22.1 millones de toneladas de basura orgánica (comida, jardines y materiales orgánicos similares). Como se sabe, cerca del 30\% del peso original de esta basura se puede transformar en composta, por lo que es viable producir cerca de 6.6 millones de toneladas de composta, que potenciarían aún más los rendimientos por hectárea del maíz de secano. Asimismo, la fabricación de compostas sería una importante cadena productiva para la creación de millones de empleos, tanto en el campo como en la ciudad.

Para transferir la innovación se propone establecer faros agroecológicos que funcionarán como vitrinas tecnológicas para promover el escalonamiento del MP-I. Se propone potenciar la transferencia del MP-I, mediante el diseño-ejecución de un modelo de comunicación alternativo al modelo de extensión convencional, basado en el método dialógico-comunicativo propuesto por Freire ${ }^{34}$. Dialógico es, en el contexto del MP-I, considerar como pares las innovaciones radicales y progresivas que interaccionan en el diálogo de saberes del MP-I.

En México no existen estadísticas oficiales sobre la superficie sembrada con milpa, pero consideramos que, al menos, el 20\% del área total sembrada (cerca de 21 millones de hectáreas) en México, se maneja como milpa en cientos de agronichos. Alcanzar la autosuficiencia alimentaria, exige identificar y aplicar un MP-I que sea ad hoc a cada uno de estos agronichos. El MP-I no puede ser sustituido por recomendaciones generales, como lo propone el INIFAP, porque simplemente no se corresponden con las condiciones concretas y generales que definen el manejo de la milpa.

La exclusión de los milperos de subsistencia de los planes sectoriales de fomento agrícola está ocasionando su proletarización o descampesinización y, por lo mismo, la mengua de sus iniciativas, capacidades, habilidades y la pérdida de las innovaciones progresivas aplicadas en el manejo de la milpa. Esta cuestión no es trivial, pues se trata de tecnologías aplicadas en el manejo de la milpa que han perdurado durante milenios y que han sido garantes de la autosuficiencia alimentaria.

\section{Reflexiones finales}

El mundo, incluido México, vive una de las crisis más profundas de los últimos tiempos que está afectando la producción y consumo de alimentos. Por esta razón, satisfacer la alimentación se proyecta para la mayoría de los milperos de secano, como la base para alcanzar una mejor vida. Esto exige aumentar su productividad, sobre todo en aquellas entidades del país que poseen bajos niveles de autosuficiencia alimentaria, y donde predominan productores que, mediante el trabajo concreto y útil, producen alimentos que en su mayoría son destinados directamente al consumo humano.

La propuesta es el MP-I, que se sustenta en la evaluación de las innovaciones radicales y progresivas que los productores aplicaron en el manejo de la milpa, que 
representa un antídoto contra la colonización del saber y la violencia epistémica.

Las evidencias halladas indican que la mayoría de los milperos de secano aplicaron el diálogo de saberes en el manejo de la milpa, la cual imita la estructura vegetacional y funcional de los ecosistemas naturales. De la cooperación que se establece entre sus elementos, derivan mayores rendimientos, la captura de gases efectos invernadero, la mitigación del cambio climático, la resiliencia y sostenibilidad. Los componentes de la milpa crean un microclima que regula la temperatura y la humedad, conservando estable su ambiente; por esta razón, la milpa puede resistir las heladas o altas temperaturas, así como las sequias o inundaciones.

Estas "nuevas" formas de manejo sostenibles están disponibles entre los productores; el reto es identificarlas y transferirlas mediante el establecimiento de faros agroecológicos para revertir los bajos rendimientos de los milperos menos eficientes y para fortalecer el abasto de maíz y la autosuficiencia alimentaria familiar de los productores más pobres.

La ejecución del MP-I tendría un impacto multisectorial: garantizará el derecho a la alimentación y nutrición, creará empleos, reducirá la emisión de gases efecto invernadero, mejorará la salud, reducirá la migración campo-ciudad y restituirá la armonía entre sociedad-naturaleza, el campo-ciudad y el tejido comunitario.

\section{Bibliografía}

WARD, Anseeuw y LAURENT, Catherine. Occupational paths towards commercial agriculture: The key roles of farm. Journal of Arid Environments, 2007, vol. 70, núm. 4, p. 659-671.

CEDRSSA (Centro de Estudios para el Desarrollo Rural Sustentable y la Soberanía Alimentaria). Reporte. El Tratado de Libre Comercio de América de Norte, el sector agroalimentario mexicano y la llegada de Donald Trump a la presidencia de los Estados Unidos. Palacio Legislativo de San Lázaro, ciudad de México. 2017. $21 \mathrm{p}$.

COCHRAN, William G. Sampling Techniques $3^{\mathrm{a}}$ ed. New York. 1977. 422 p.

DAMIÁN-HUATO, Miguel Ángel. Proyecto de investigación: "Faros agroecológicos y seguridad alimentaria para productores de maíz de secano en Jolalpan, Puebla-México." Registro: DITCo2015-7, Reporte de Resultados del Proyecto de Innovación Tecnológica-BUAP. 2015. México. 4 p.

DE GRAMMONT, Hubert. La nueva estructura ocupacional en los hogares rurales mexicanos. En: De Grammont H., Martínez L. (Coord.). La pluriactividad en el campo mexicano. FLACSO. Ecuador. 2009. p. 273-303.

DE SOUSA, Santos. Renovar la teoría crítica y reinventar la emancipación social. "Encuentros en Buenos Aires". CLACSO. Buenos Aires. 2006. 110 p.

FAO (Organización de las Naciones Unidas para la Alimentación y la Agricultura). El estado mundial de la agricultura y la alimentación, cambio climático, agricultura y seguridad alimentaria. Consultado el 15 de enero de 2018. http://www.fao. org/3/a-i6030s.pdf. 
FERRERA-CERRATO, Ronald., ALARCÓN, Alejandro. La microbiología del suelo en la agricultura sostenible. Ciencia Ergo Sum, 2001, vol. 8, núm 1. p. 175-183.

FLORES, Claudia Cecilia, SARANDÓN, Santiago Javier. Desarrollo y evolución de los ecosistemas. En: Sarandón, S.J. Flores, C.C (ed.) Agroecología: Bases teóricas para el diseño y manejo de aes sustentables. 2014. Facultad de Ciencias Agrarias y Forestales. Argentina. p. 159-189.

FREIRE, Paulo. ¿Extensionismo o Comunicación? Siglo XXI (ed), 1973. México. 85 p. GALENDE, J. (2008). La organización del proceso de innovación en la empresa española. Revista economía industrial, Núm. 368, Ministerio de Industria, Energía y Turismo. ISSN 0422-2784, España.

GONZÁLEZ-ORTEGA, Enrique, PIÑEYRO-NELSON, Angel, GÓMEZ HERNÁNDEZ, Eduardo, MONTERRUBIO-VÁZQUEZ, Ernesto. DÁVILA-VELDERRAIN, Arleo, ÁLVAREZ-BUYLLA, E. Pervasive presence of transgenes and glyphosate in maize derived food in Mexico. Agroecology and Sustainable Food Systems. 2017, vol. 41, núm. 9 and 10, p. 1146-1161. DOI: 10.1080/21683565.2017.1372841.

HERRERA, J, CADENA, P, SAN-CLEMENTE, A. Diversidad de la artropodofauna en monocultivo y policultivo de maíz (Zea mays) y habichuela (Phaseolus vulgaris). 2005. Boletín del Museo de entomología de la Universidad del Valle, vol. 6, núm. 1, p. 23-31.

HOLT, G. Campesino a campesino, Managua Nicaragua, Voces de Latinoamérica Movimiento Campesino para la Agricultura Sustentable. 2008. 294 p.

INIFAP. Paquete tecnológico para el cultivo de maíz, Distrito de Desarrollo Rural de Cholula e Izúcar de Matamoros, Puebla-México. 2009. Base de datos CD-ROM.

MAGALHANES, A, DA SILVA, W. Determinantes genético-fisiológicos da produtividade do milho: melhoramiento y produgáo do milho. 1987. 425 p.

MANUAL DE OSLO (2006). Guía para la recogida e interpretación de datos sobre innovación. Tercera edición. Organización de Cooperación y Desarrollo Económico y Oficina de Estadísticas de las Comunidades Europeas, España. 168p.

MIGNOLO, W. Desobediencia Epistémica: Retórica de la modernidad, lógica de la colonialidad y gramática de la descolonialidad. Editorial del Signo, Argentina. 2010. 126 p.

NICOLESCU, Basarab. La Transdisciplinariedad. Manifiesto: Multiversidad Mundo Real. México. 2009. 206 p.

ONU (Organización de las Naciones Unidas). Asamblea General de Naciones Unidas, en su resolución 217 A (III). 1948, Artículo 25, p.6. http://www.un.org/es/rights/ overview/.

PATROUILLEAU, M. M. Lisandro Martínez, Eduardo Cittadini y Roberto Cittadini (2017). Políticas públicas y desarrollo de la agroecología en Argentina. En: Políticas Públicas a favor de la Agroecología en América Latina y el Caribe. Red Políticas Publicas en América Latina y el Caribe (PP-AL), Brasilia, Brasil.

PIAGET, J., R. García. (2008). Psicogénesis e historia de la ciencia, editorial Siglo XXI, Undecima edición. 2008. 252 p. 
PLOEG, J. D. 2010. Nuevos campesinos e imperios alimentarios. Icaria, editorial. Barcelona, España.

PRETTY, J, TOULMIN, C, WILLIAMS, S. Sustainable intensification in African agricultura. 2011. International journal of agricultural sustainability. vol. 9, núm. 1, p. 5-24.

ROBERT, Michel. Captura de carbono en los suelos para un mejor manejo de la Tierra. 2002. ONU. Institut national de recherche agronomique, París, Francia. 61 p.

RUBIO, Blanca. El dominio del hambre. Crisis de hegemonía y alimentos. Universidad Autónoma Chapingo, Colegio de Posgraduados, Universidad de Zacatecas. 2014, México, 270 p.

SIAP (Servicio de Información Agroalimentaria y Pesquera) (2017). Series históricas de superficie sembrada y cosechada, 1980-2014, (en línea). http://www.siap.gob. $\mathrm{mx} /$ index.php?option=com_wrapper\&view=wrapper\&Itemid=351, 7 de enero de 2013.

SNIARN (Sistema Nacional de Información Ambiental y de Recursos Naturales) (2015), Secretaría de Medio Ambiente y Recursos Naturales (SEMARNAT), consulta temática. Residuos sólidos urbanos. Disponible en: http://dgeiawf.semarnat. gob.mx:8080/approot/dgeia_mce/html/mce_index.html.(accesado el 30 de enero de 2015).

TOLEDO, V. M. (2005). La memoria tradicional: la importancia agroecológica de los saberes locales. Leisa, vol. 20, núm. 4, p. 16-19.

WALKER, B. Biodiversity and ecological redundancy. 1992. Conservation Biology, vol. 6, p. 18-23.

WARMAN, Arturo. El campo mexicano en el siglo XX. 2001. Fondo de cultura económica, México. 262 p.

ZEMELMAN, Hugo. La totalidad como perspectivas de descubrimiento. 1987. Revista Mexicana de Sociología, vol. 49, núm. 1, p. 13-31.

ZEMELMAN, Hugo. Los horizontes de la razón I: dialéctica y apropiación del presente. 1992. Barcelona. Anthropos. 255 p.

(c) Copyright: Miguel Ángel Damián Huato, Artemio Cruz León, Dora Ma. Sangerman-Jarquín, Lucía López Reyes, Moisés Carcaño Montiel y Omar Romero Arenas, 2019

(c) Copyright: Scripta Nova, 2019

Ficha bibliográfica:

DAMIÁN HUATO, Miguel Ángel; CRUZ LEÓN, Artemio; SANGERMAN-JARQUÍN, Dora Ma.; LÓPEZ REYES, Lucía; CARCAÑO MONTIEL, Moisés; ROMERO ARENAS, Omar; Modelo productor-innovador y autosuficiencia alimentaria para milperos de secano: propuesta de política pública sostenible, Scripta Nova. Revista Electrónica de Geografía y Ciencias Sociales. Barcelona: Universitat de Barcelona, 15 de febrero de 2019, vol. XXIII, nº 608. [ISSN: 1138-97] 